\title{
BMJ Open Scoping review protocol on maternal, newborn and child health research in Ethiopia
}

\author{
Grace J Chan (D) , ${ }^{1,2}$ Misrak Getnet, ${ }^{3}$ Ronke Olowojesiku, ${ }^{2}$ Thein Min-Swe, ${ }^{2}$ \\ Bezawit Hunegnaw, ${ }^{4}$ Delayehu Bekele ${ }^{5}$
}

To cite: Chan GJ, Getnet M, Olowojesiku R, et al. Scoping review protocol on maternal, newborn and child health research in Ethiopia. BMJ Open 2020;10:e034307. doi:10.1136/ bmjopen-2019-034307

- Prepublication history and additional material for this paper are available online. To view these files, please visit the journal online (http://dx.doi. org/10.1136/bmjopen-2019034307).

Received 13 September 2019 Revised 06 March 2020 Accepted 20 May 2020

\section{Check for updates}

(c) Author(s) (or their employer(s)) 2020. Re-use permitted under CC BY-NC. No commercial re-use. See rights and permissions. Published by BMJ.

${ }^{1}$ Department of Pediatrics, Harvard Medical School, Boston, Massachusetts, USA

${ }^{2}$ Department of Epidemiology, Harvard University T.H. Chan

School of Public Health, Boston, Massachusetts, USA

${ }^{3}$ Health Systems Directorate, Ethiopian Public Health Institute, Addis Ababa, Ethiopia

${ }^{4}$ Department of Pediatrics, Saint Paul's Hospital Millennium Medical College, Addis Ababa, Ethiopia

${ }^{5}$ Department of Obstetrics and Gynecology, Saint Paul's Hospital Millennium Medical College, Addis Ababa, Ethiopia

Correspondence to

Dr Grace J Chan;

grace.chan@hsph.harvard.edu

\section{ABSTRACT}

Introduction There has been a tremendous reduction in maternal and child mortality in the last decade. However, a significant number of deaths still occur disproportionately in low-income country settings. Ethiopia is the secondmost populous nation in sub-Saharan Africa with a high maternal mortality rate of 412 deaths per 100000 live births and an under-five mortality rate of 55 per 1000 live births. This study presents a scoping review protocol to describe the current knowledge of maternal and child health in Ethiopia to identify gaps for prioritisation of future maternal, newborn and child health research.

Methods and analyses A search strategy will be conducted in PubMed/MEDLINE, EMBASE and the WHO African Index Medicus. Researchers will independently screen title and abstracts followed by full texts for inclusion. Study characteristics, research topics, exposures and outcomes will be abstracted from articles meeting inclusion criteria using standardised forms. Descriptive analysis of abstracted data will be conducted.

Ethics and dissemination Data will be abstracted from published manuscripts and no additional ethical approval is required. The results of the review will be shared with maternal and child health experts in Ethiopia through stakeholder meetings to prioritise research questions. Findings will be submitted to a peer-reviewed journal for publication, in addition to national-level and global-level disseminations.

\section{INTRODUCTION}

Globally, there have been significant improvements in reducing maternal and under-five mortality. ${ }^{1}$ Since 2000 , there has been a $37 \%$ reduction in the maternal mortality rate, and the under-five mortality rate has reduced almost by half. ${ }^{1}$ However, of the estimated 300000 maternal deaths that occurred worldwide in 2015, approximately $99 \%$ occurred in developing countries. ${ }^{2}$ With 11000 maternal deaths each year and a maternal mortality rate of $412 / 100000$ live births, ${ }^{2}{ }^{3}$ Ethiopia is one of the six countries that has contributed to more than half of the global maternal deaths. ${ }^{4}$ With an estimated 80000 newborn deaths each year in Ethiopia (neonatal mortality rate $30 / 1000$ live births) ${ }^{5}$ Ethiopia

\section{Strengths and limitations of this study}

To our knowledge, this will be the first scoping review describing the landscape of maternal, newborn and child health $(\mathrm{MNCH})$ research in Ethiopia.

- Findings from the review will be shared with stakeholders in-country for decision-making and priority setting.

- The scoping review will be limited to published data, which will affect publication bias.

- The scoping review covers a broad search strategy to present an overview of MNCH research in Ethiopia. To answer specific research questions, further work can be conducted through systematic reviews and meta-analyses.

is one of the five countries accounting for half of Africa's newborn deaths each year. ${ }^{6}$

These figures highlight the work that remains to improve maternal, newborn and child health $(\mathrm{MNCH})$ in countries which account for the greatest number of maternal and child deaths. In Africa's second-most populous nation, Ethiopia, many women still die from preventable causes such as maternal haemorrhage and infection. Ethiopia was successful in achieving Millennium Development Goal 4 for a two-thirds reduction in under-five mortality through a combination of efforts in health, nutrition and non-health sectors. $^{7-9}$ Under-five mortality improved from 166/1000 live births in 2000 to 55/1000 live births in 2019. ${ }^{3} 5$ However, newborn mortality remains one of the highest in the world at 30 deaths per 1000 live births. ${ }^{5}$ Data on stillbirths remain limited.

To determine research priorities, more information is needed to describe what is currently known and what the gaps are in the maternal and child health literature in countries like Ethiopia. This will be the first scoping review in Ethiopia describing the broad, heterogeneous and complex nature of MNCH research. 


\section{Study objectives}

The main aims of this scoping review are:

1. To determine the study characteristics, study types and designs (eg, cohort, randomised control trial) and content (ie, exposures, outcomes, interventions) during the preconception period, pregnancy, birth, postpartum, motherhood and childhood for $\mathrm{MNCH}$ research in Ethiopia.

2. To identify gaps in the existing literature on $\mathrm{MNCH}$ research in Ethiopia.

3. To inform on priority research questions for primary data collection related to $\mathrm{MNCH}$ in Ethiopia.

\section{METHODS AND ANALYSIS}

We will conduct a scoping review of all published literature using the framework proposed by Arksey and O'Malley and expanded on by Levac et al and the Joanna Briggs Institution (JBI) ${ }^{10-12}$ The following stages are (1) identification of the research question, (2) identification of relevant studies, (3) study selection, (4) data charting, (5) collation, summarisation and report of results and (6) consultation with key stakeholders to communicate and discuss findings from the review. This scoping review will use the Preferred Reporting Items for Systematic reviews and Meta-Analyses extension for Scoping Reviews (PRISMA-ScR) checklist as a guide. ${ }^{13}$

\section{Patient and public involvement}

Patients and the public were not involved in the development of the protocol.

\section{Stage 1: identifying a research question}

The research team, in consultation with in-country stakeholders, identified broad, comprehensive research questions through an iterative process. The following questions will be addressed by this protocol: what types of studies, study questions and data on exposures, outcomes and interventions have been studied in $\mathrm{MNCH}$ research in Ethiopia?

\section{Stage 2: identifying relevant studies}

To identify relevant literature, the study team will develop a comprehensive search strategy (online supplementary appendix 1). Studies published in English or Amharic will be included. The team will search PubMed/MEDLINE, EMBASE and the African Index Medicus via the Global Index Medicus platform of the WHO. The search will not be restricted by time to describe the volume of literature published on MNCH in Ethiopia through January 2019. References will be downloaded and imported into a reference library using EndNote version x9.

Inclusion criteria will use the population, concepts and context framework described by JBI, shown in table $1 .^{12}$

On study type, we plan to include all evidence-based studies or studies that apply "principles of scientific reasoning, including systematic uses of data and information systems and appropriate use of behavioural science theory and programme planning models', ${ }^{14}$ in
Table 1 Inclusion criteria using population, concepts and context framework

\begin{tabular}{|c|c|}
\hline Population & $\begin{array}{l}\text { Women of reproductive age (women 15-49 } \\
\text { years). } \\
\text { Pregnant women regardless of age. } \\
\text { Postpartum women (birth to } 42 \text { days } \\
\text { postpartum). } \\
\text { Mothers of children under } 5 \text { years. } \\
\text { Newborns ( } 28 \text { days or less). } \\
\text { N Infants ( } 29 \text { days to under } 1 \text { year). } \\
\text { - Children ( } 1 \text { year to under } 10 \text { years). }\end{array}$ \\
\hline Concepts & $\begin{array}{l}\text { Preconception care defined by WHO as } \\
\text { 'the provision of biomedical, behavioural } \\
\text { and social health interventions before } \\
\text { conception occurs'. }{ }^{\text {. }} \\
\text { Reproductive health defined by National } \\
\text { Library of Medicine (NLM) definition as the } \\
\text { state of optimal female reproductive system } \\
\text { functioning in the absence of disease, } \\
\text { disorders or deficiencies. } \\
\text { Maternal health defined by NLM as the } \\
\text { health of women during pregnancy, } \\
\text { childbirth and the postpartum period. } \\
\text { Newborn health defined as the physical } \\
\text { and mental wellness of individuals under } 28 \\
\text { days of age. } \\
\text { Infant health defined as the physical and } \\
\text { mental wellness of individuals under } 1 \text { year } \\
\text { of age. } \\
\text { Child health defined as the physical and } \\
\text { mental wellness of individuals under } 10 \\
\text { years of age. }\end{array}$ \\
\hline Context & $\begin{array}{l}\text { Ethiopian studies. }{ }^{\dagger} \\
\text { - Time-since } 1946 .^{\ddagger}\end{array}$ \\
\hline
\end{tabular}

"The WHO definition of adolescence is individuals aged 10-19. Children are considered less than 10 for the purposes of this review.

${ }^{\dagger}$ Ethiopian studies will be defined as studies conducted in-country and with non-refugee populations.

${ }_{1} 1946$ is the earliest date of coverage across all databases used in the review.

peer-reviewed published literature that are accessible online and through interlibrary requests. Such studies could include randomised trials, observational studies, physiologic studies, case studies, laboratory studies, systematic reviews and meta-analyses. ${ }^{15-18}$ Review of grey literature will be outside the scope of this review.

Non-English or non-Amharic, non-human, personal opinion pieces, non-systematic literature reviews and non-journal articles will be excluded. To maintain focus, we excluded sex workers, non-Ethiopian refugee populations and children above the age of 10 . We will screen for these articles and count the number of studies related to these populations to understand potential biases associated with excluding these populations.

\section{Stage 3: study selection}

Study selection forms will be developed and piloted on a random sample of references derived from search results 
by two independent reviewers. The kappa statistic will be used to measure the agreement between reviewers. Disagreement will be discussed between reviewers and refinement of screening form will be made based on discussion. Edits to the pilot forms will be made based on discussions to resolve disagreements between reviewers on a random sample of references until reviewer agreement of 0.8 or greater (as determined by kappa statistic) is achieved.

Once forms are finalised, two research assistants will independently screen references for relevance to research question using the inclusion and exclusion criteria described in stage 2 . Study selection will be conducted in two phases: title and abstract screen and full-text review. Any disagreements will be resolved by consensus between reviewers. If consensus cannot be reached, a third reviewer will serve as a tie-breaker.

\section{Title and abstract screen}

After removal of duplicates, titles and abstracts will be screened for full-text review by two independent reviewers. During the title and abstract screen, each study will be classified as: 'yes', 'no' or 'unclear'. All studies marked as 'yes' or 'unclear' will be included for full-text review. For excluded studies, basis of exclusion will be documented in review.

\section{Full-text review}

Articles selected for full-text review in which the full text is unavailable will be documented. All available full-text articles will be screened for inclusion by two independent reviewers. Screening at this stage will use the same inclusion and exclusion criteria used for the title and abstract screen. Should any questions/concerns arise about a particular study, an attempt will be made to contact the authors of the study.

\section{Stage 4: data charting}

As per Arksey and O'Malley, data charting 'describes a technique for synthesising and interpreting qualitative data by sifting, charting and sorting material according to key issues and themes'. ${ }^{10}$ Once screening is complete, a data extraction tool will be developed in Qualtrics to chart results of review. The tool will be piloted and refined.

Data will be abstracted from full-text papers that meet inclusion criteria. We will collect available data on author(s), publication year, journal of publication, study population, sample size, type of study/study design, ${ }^{15-18}$ duration in months, type of study question, region of study (eg, rural vs urban, specific district), setting of study (eg, academic, community-based, laboratory-based), country of corresponding author, funding source. For epidemiologic studies, we will collect the following data: types of interventions, exposures and outcomes. These categories are further described in online supplementary appendix $2{ }^{19-26}$ For other studies, the study aim will be abstracted and thematically coded by research topic.
Data will be collected independently by two reviewers and reconciled between the reviewer pairs. Any discrepancies in extraction will be resolved by consensus between the reviewers. If consensus cannot be reached by two reviewers, a third reviewer will resolve the disagreement. Agreement between reviewer pairs will be documented. To ensure inter-reviewer reliability, the team coordinator will review a random sample of articles and provide feedback to improve standardisation across reviewer pairs.

\section{Stage 5: collation, summarisation and report of results}

Studies will be collated and summarised by years of publication, journals of publication, number of studies included, number of studies by $\mathrm{MNCH}$ population, number of studies by research topic and types of studies, and types of interventions, exposures and outcomes by specific MNCH populations. An assessment of quality will not be conducted.

We will conduct descriptive analysis and present the results using graphics and tables. Adjustment to data reporting scheme will be made as needed based on findings. We will conduct the scoping review following the PRISMA-ScR checklist. ${ }^{13}$

\section{ETHICS AND DISSEMINATION}

Throughout the process of this review, we will involve key stakeholders in-country such that the final conclusions are reflective of the MNCH work being done in Ethiopia. The scoping review is conducted with the Ethiopian Public Health Institute, Ministry of Health, St. Paul's Hospital Millennium Medical College, and Harvard T.H. Chan School of Public Health. Authors have an established long-term working relationship on completed and ongoing maternal and child health studies. Data from the review will be summarised and presented during stakeholder meetings with collaborators in Ethiopia to prioritise $\mathrm{MNCH}$ research questions. A final report will be developed and disseminated through a peer-reviewed journal.

\section{CONCLUSIONS}

We have described a protocol for a scoping review on $\mathrm{MNCH}$ research in Ethiopia. This scoping review will contribute to the $\mathrm{MNCH}$ field by examining literature to map study topics, to describe study characteristics and populations and to identify research gaps. In particular, the review will identify understudied populations and $\mathrm{MNCH}$ research topics to develop future research questions. Depending on the availability of data and potential for impact, systematic reviews and meta-analyses can be conducted to further summarise the findings on specific research questions. To our knowledge, this review is the first of its kind to be done on the subject. By providing a broad overview of the MNCH literature, this review will prioritise research questions to improve maternal and child health in Ethiopia. 
Contributors All authors contributed to the preparation of the manuscript. The specific contributions are listed below: conceptualisation of the study and first drafting of the manuscript: GC, MG and RO. Subsequent revisions of manuscript drafts, completion of information on study settings and methods: GC, MG, TM-S, BH and DB. All authors read and approved the final manuscript.

Funding This work is supported by the Bill \& Melinda Gates Foundation.

Competing interests None declared.

Patient and public involvement Patients and/or the public were not involved in the design, or conduct, or reporting or dissemination plans of this research.

Patient consent for publication Not required.

Provenance and peer review Not commissioned; externally peer reviewed.

Open access This is an open access article distributed in accordance with the Creative Commons Attribution Non Commercial (CC BY-NC 4.0) license, which permits others to distribute, remix, adapt, build upon this work non-commercially, and license their derivative works on different terms, provided the original work is properly cited, appropriate credit is given, any changes made indicated, and the use is non-commercial. See: http://creativecommons.org/licenses/by-nc/4.0/.

ORCID iD

Grace J Chan http://orcid.org/0000-0002-2716-1643

\section{REFERENCES}

1 Nations U. The sustainable development goals report, 2018.

2 World Health Organization. Trends in maternal mortality 1990 to 2015: estimates by WHO, UNICEF, UNFPA, world bank group and the United nations population division. Geneva, Switzerland: World Health Organization, 2015.

3 USAID. Demographic and health survey 2016. Maryland: Central Statistical Authority and ORC Macro, 2017

4 Hogan MC, Foreman KJ, Naghavi M, et al. Maternal mortality for 181 countries, 1980-2008: a systematic analysis of progress towards millennium development goal 5. Lancet 2010;375:1609-23.

5 Federal Ministry of Health, The DHS Program ICF. Ethiopia minidemographic and health survey. Addis Ababa: Ethiopian Public Health Institute, 2019.

6 Joy Lawn PM, Cousen S. Africa's newborns-counting them and making them count, 2019. Available: https://www.who.int/pmnch/ media/publications/aonsection I.pdf [Accessed 21 Aug 2019].

7 Assefa Y, Damme WV, Williams OD, et al. Successes and challenges of the millennium development goals in Ethiopia: lessons for the sustainable development goals. BMJ Glob Health 2017;2:e000318.
8 Moucheraud C, Owen H, Singh NS, et al. Countdown to 2015 country case studies: what have we learned about processes and progress towards MDGs 4 and 5? BMC Public Health 2016;16:794.

9 Ruducha J, Mann C, Singh NS, et al. How Ethiopia achieved millennium development goal 4 through multisectoral interventions: a countdown to 2015 case study. Lancet Glob Health 2017;5:e1142-51.

10 Arksey H, O'Malley L. Scoping studies: towards a methodological framework. Int J Soc Res Methodol 2005;8:19-32.

11 Levac D, Colquhoun H, O'Brien KK. Scoping studies: advancing the methodology. Implement Sci 2010;5:69.

12 Peters MDJ, Godfrey CM, Khalil H, et al. Guidance for conducting systematic scoping reviews. Int J Evid Based Healthc 2015;13:141-6.

13 Tricco AC, Lillie E, Zarin W, et al. PRISMA extension for scoping reviews (PRISMA-ScR): checklist and explanation. Ann Intern Med 2018;169:467-73.

14 Brownson RC, Baker EA, Deshpande AD, et al. Evidence-based public health. Oxford University Press, 2017.

15 Creswell JW, Clark VLP. Designing and conducting mixed methods research. Sage publications, 2017.

16 Berg B L. Qualitative research methods for the social sciences, 2001.

17 Rothman KJ, Greenland S, Lash TL. Modern epidemiology: wolters kluwer health. Philadelphia: Lippincott Williams \& Wilkins, 2012.

18 University GW. Study design 101, 2019. Available: https:// himmelfarb.gwu.edu/tutorials/studydesign101

19 Organization WH. Meeting to develop a global consensus on preconception care to reduce maternal and childhood mortality and morbidity. Geneva: World Health Organization Headquarters, 2012.

20 HP. Social determinants of health, 2020. Available: https://www. healthypeople.gov/2020/topics-objectives/topic/social-determinantsof-health

21 (IHME) IfHMaE. GBD compare data visualization. Seattle, WA: IHME, University of Washington, 2018. http://vizhub.healthdata.org/gbdcompare

22 Hunegnaw B. Top maternal conditions based on HMIS ranking data for Ethiopia. Addis Ababa, Ethiopia, 2018.

23 Hunegnaw B. Top pediatric conditions based on HMIS ranking data for Ethiopia. Addis Ababa, Ethiopia, 2018.

24 Organization WH. The WHO application of ICD-10 to deaths during pregnancy, childbirth and puerperium: ICD-MM. World Health Organization, 2012.

25 Organization WH. The WHO application of ICD-10 to deaths during the perinatal period: ICD-PM, 2016.

26 The Partnership for Maternal N, \& Child Health. A global review of the key interventions related to reproductive, maternal, newborn and child health (RMNCH). Geneva, Switzerland: PMNCH, 2011. 